Document downloaded from:

http://hdl.handle.net/10251/107461

This paper must be cited as:

Bernardes, NCJ.; Peris Manguillot, A.; Ródenas Escribá, FDA. (2017). Set-Valued Chaos in Linear Dynamics. Integral Equations and Operator Theory. 88(4):451-463. doi:10.1007/s00020-017-2394-6

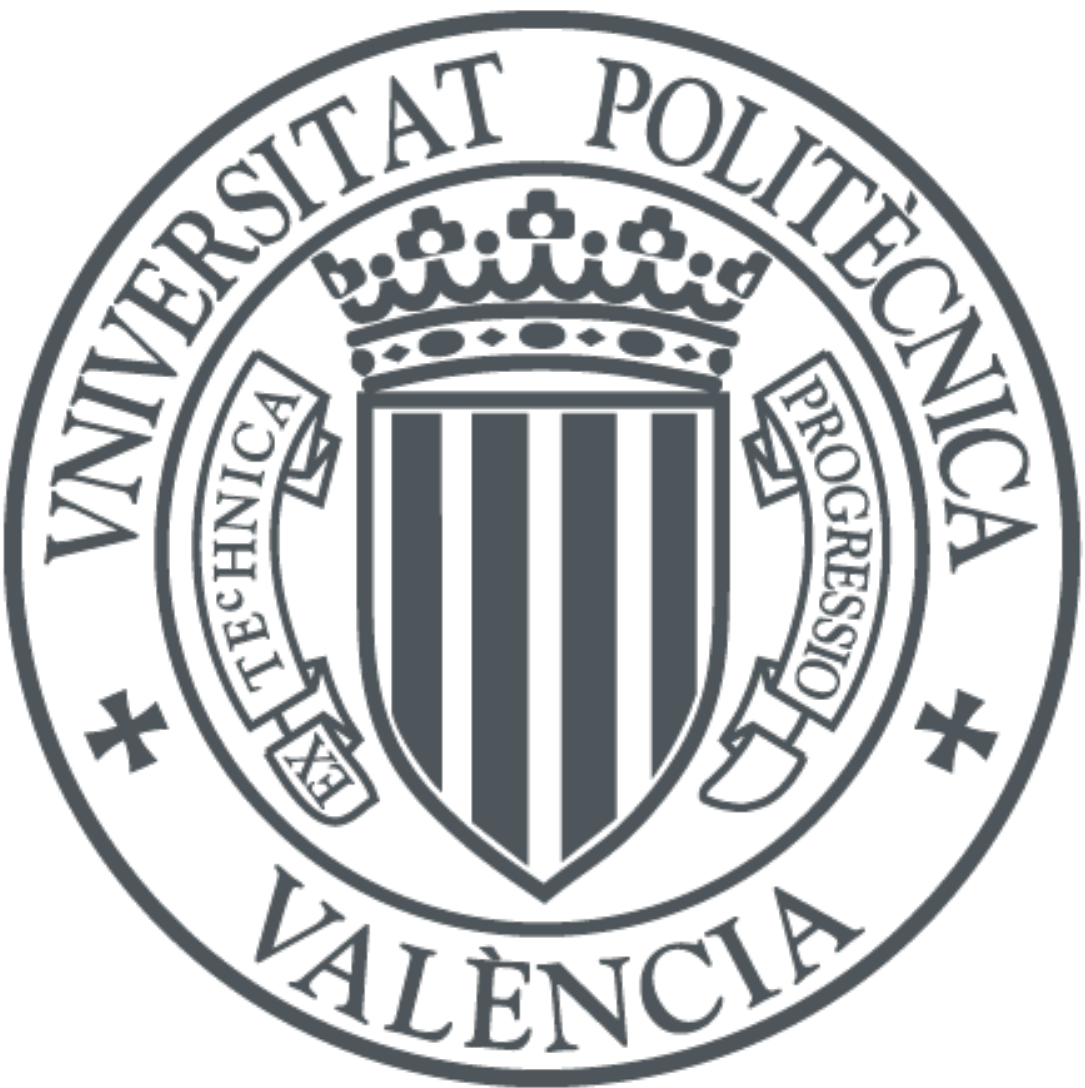

The final publication is available at

http://doi.org/10.1007/s00020-017-2394-6

Copyright Springer-Verlag

Additional Information 


\title{
Set-Valued Chaos in Linear Dynamics
}

\author{
N. C. Bernardes Jr., A. Peris and F. Rodenas
}

\begin{abstract}
We study several notions of chaos for hyperspace dynamics associated to continuous linear operators. More precisely, we consider a continuous linear operator $T: X \rightarrow X$ on a topological vector space $X$, and the natural hyperspace extensions $\bar{T}$ and $\widetilde{T}$ of $T$ to the spaces $\mathcal{K}(X)$ of compact subsets of $X$ and $\mathcal{C}(X)$ of convex compact subsets of $X$, respectively, endowed with the Vietoris topology. We show that, when $X$ is a complete locally convex space (respectively, a locally convex space), then Devaney chaos (respectively, topological ergodicity) is equivalent for the maps $T, \bar{T}$ and $\widetilde{T}$. Also, under very general conditions, we obtain analogous equivalences for Li-Yorke chaos. Finally, some remarks concerning the topological transitivity and weak mixing properties are included, extending results in [1] and [30].
\end{abstract}

Mathematics Subject Classification (2010). Primary 47A16; Secondary 37B99.

Keywords. Hyperspace dynamics, linear dynamics, Devaney chaos, LiYorke chaos, mixing properties.

\section{Introduction}

An interesting topic in the area of dynamical systems is the comparison between individual dynamics (the action of the system on points of the phase space) and collective dynamics (the action of the system on subsets of the phase space). Consider the action of a continuous map $f: X \rightarrow X$ on a topological space $X$. The most usual context for collective dynamics is that of the induced map $\bar{f}$ on the hyperspace of all nonempty compact subsets of $X$ endowed with the Vietoris topology. The first study about the connection between dynamical properties of the dynamical system generated by the map $f$ and the induced system generated by $\bar{f}$ on the hyperspace was given by

The first author was partially supported by CNPq (Brazil) and by the EBW + Project (Erasmus Mundus Programme). The second and third authors were supported by MINECO, Projects MTM2013-47093-P and MTM2016-75963-P. The second author was partially supported by GVA, Project PROMETEOII/2013/013. 
Bauer and Sigmund [2] in 1975. Since this work, the subject of hyperspace dynamical systems has attracted the attention of many researchers.

Román-Flores [32] and Fedeli [12] studied the relation of chaos for discrete dynamical systems with the corresponding hyperspace version. Banks [1], Liao, Wang and Zhang [27], and Peris [30] solved independently the main problem proposed in $[32,12]$ by giving a characterization of topological transitivity for collective dynamics in terms of the weak mixing property for individual dynamics. Several recent results in this topic can be found in, e.g., $[25,36,18,28,37,24,13,19,29,7,26,39]$.

In the context of linear dynamics (see the books $[4,17]$ for a thorough study of this topic), the interest on collective dynamics was initiated by Herzog and Lemmert [20] in 1998, who obtained a sufficient condition for the existence of a dense orbit under the induced map. Further results were obtained by Peris [30] in 2005 in connection to the Hypercyclicity Criterion. In [38] Wu, Xue and Ji studied the hyperspace linear dynamics for connected compact sets. Our goal here is to continue this line of investigation by studying several notions of chaotic behaviour, including Devaney chaos, Li-Yorke chaos, topological ergodicity and mixing properties.

Let us now recall some basic definitions and notations for hyperspace dynamics. Given a topological space $X$, we denote by $\mathcal{K}(X)$ the hyperspace of all nonempty compact subsets of $X$ endowed with the Vietoris topology, that is, the topology whose basic open sets are the sets of the form

$$
\mathcal{V}\left(U_{1}, \ldots, U_{r}\right):=\left\{K \in \mathcal{K}(X): K \subset \bigcup_{i=1}^{r} U_{i} \text { and } K \cap U_{i} \neq \emptyset, \quad i=1, \ldots, r\right\},
$$

where $r \geq 1$ and $U_{1}, \ldots, U_{r}$ are nonempty open subsets of $X$. When the topology of $X$ is induced by a metric $d$, the topology of $\mathcal{K}(X)$ is induced by the Hausdorff metric associated to $d$, namely

$$
d_{H}\left(K_{1}, K_{2}\right):=\max \left\{\max _{x_{1} \in K_{1}} d\left(x_{1}, K_{2}\right), \max _{x_{2} \in K_{2}} d\left(x_{2}, K_{1}\right)\right\} .
$$

If $T: X \rightarrow X$ is a continuous map, then $\bar{T}: \mathcal{K}(X) \rightarrow \mathcal{K}(X)$ denotes the induced map defined by

$$
\bar{T}(K):=T(K) \text { for } K \in \mathcal{K}(X),
$$

where $T(K):=\{T x: x \in K\}$ as usual. Note that $\bar{T}$ is also continuous. We refer the reader to the book [23] for a detailed study of hyperspaces.

In the present work we are interested in the case where $X$ is a topological vector space and $T: X \rightarrow X$ is a continuous linear operator. In this context, we also consider the $\operatorname{set} \mathcal{C}(X)$ of all convex elements of $\mathcal{K}(X)$ endowed with the topology induced by that of $\mathcal{K}(X)$ and the continuous map $\widetilde{T}: \mathcal{C}(X) \rightarrow \mathcal{C}(X)$ obtained by restricting $\bar{T}$ to $\mathcal{C}(X)$. Additionally, we will need the following auxiliary families of compact subsets of $X$ : the set $\mathcal{F}(X)$ of all finite subsets of $X$ and the set $\mathcal{F C}(X)$ of all finite-dimensional elements of $\mathcal{C}(X)$. It is clear that $\bar{T}(\mathcal{F}(X)) \subset \mathcal{F}(X)$ and $\bar{T}(\mathcal{F C}(X)) \subset \mathcal{F C}(X)$. It is worthwhile to point out that, although the dynamics of linear operators is mainly studied when 
the phase space $X$ is a metrizable, complete and separable topological vector space, several of our results are stated and valid for operators on more general topological vector spaces, which also deserve attention (see, e.g., [10, 35] and Chapter 12 in [17]).

The paper is organized as follows: Devaney chaos (respectively, topological ergodicity) is analyzed in Section 2 for hyperspace dynamics associated to operators on complete locally convex spaces (respectively, on locally convex spaces), obtaining the corresponding equivalences for the maps $T, \bar{T}$ and $\widetilde{T}$. We also consider the general framework of $\mathcal{A}$-transitivity for a proper filter $\mathcal{A}$. In Section 3, under very general conditions, we obtain analogous equivalences for Li-Yorke chaos. This result is illustrated for the particular case of weighted shifts. Section 4 contains some remarks on topological transitivity and weak mixing properties of $T, \bar{T}$ and $\widetilde{T}$, extending results in [1] and [30].

\section{Devaney chaos and topological ergodicity}

The main result in this section is the equivalence of Devaney chaos for a single operator $T$ and its associated hyperspace maps $\bar{T}$ and $\widetilde{T}$. We should emphasize that the corresponding equivalence does not hold in general for the non-linear setting.

First, let us recall some definitions. Given a topological space $X$ and a continuous map $T: X \rightarrow X$, recall that $T$ is said to be topologically transitive (respectively, mixing) if, for any pair $U, V \subset X$ of nonempty open sets, there exists $n \geq 0$ (respectively, $n_{0} \geq 0$ ) such that $T^{n}(U) \cap V \neq \emptyset$ (respectively, for all $n \geq n_{0}$ ). Moreover, $T$ is said to be weakly mixing if $T \times T$ is topologically transitive on $X \times X$. If, given $U, V \subset X$, we set

$$
N_{T}(U, V):=\left\{n \in \mathbb{Z}_{+}: T^{n}(U) \cap V \neq \emptyset\right\},
$$

then transitivity (respectively, mixing) is equivalent to the property that $N_{T}(U, V)$ is nonempty (respectively, co-finite) for each pair of nonempty open sets $U, V \subset X$. The weak mixing property means that $N_{T}\left(U_{1}, V_{1}\right) \cap$ $N_{T}\left(U_{2}, V_{2}\right) \neq \emptyset$ for any nonempty open sets $U_{1}, V_{1}, U_{2}, V_{2} \subset X$.

In the case $X$ is a topological vector space and $T: X \rightarrow X$ is a continuous linear operator, recall that $T$ is said to be hypercyclic if there exists a vector $x \in X$ whose $T$-orbit $\operatorname{Orb}(x, T):=\left\{x, T x, T^{2} x, \ldots\right\}$ is dense in $X$. In general, hypercyclicity is a stronger notion than topological transitivity, but these two notions coincide for operators on separable $F$-spaces by Birkhoff's transitivity theorem (see [17], Theorem 1.16). We also recall that an operator $T$ is said to be Devaney chaotic if $T$ is topologically transitive and has a dense set of periodic points. In the sequel, $\operatorname{Per}(T)$ will denote the set of all periodic points of $T$.

The following lemma will be a useful tool in the paper. It is partially inspired by an argument in [20]. We recall that the closed convex hull of a set $A \subset X$ is the closure of the set of all convex combinations of finite families of elements in $A$, and it is denoted by $\overline{\mathrm{co}}(A)$. If $A$ is finite, then $\overline{\mathrm{co}}(A)$ is compact 
and finite-dimensional. If $X$ is a complete locally convex space, then $\overline{\mathrm{co}}(K)$ is compact whenever $K \subset X$ is compact ([33], Theorem 3.20(c)).

Lemma 2.1. Let $T$ be a continuous linear operator on a locally convex space $X$, and let $S: \mathcal{K}(X) \rightarrow 2^{X}$ be defined as $S(K):=\overline{\mathrm{co}}(K), K \in \mathcal{K}(X)$. Then $\left.S\right|_{\mathcal{F}(X)}: \mathcal{F}(X) \rightarrow \mathcal{C}(X)$ is a continuous map with dense range and $\left.\left.S\right|_{\mathcal{F}(X)} \circ \bar{T}\right|_{\mathcal{F}(X)}=\left.\widetilde{T} \circ S\right|_{\mathcal{F}(X)}$. Moreover, if $X$ is complete, then $S: \mathcal{K}(X) \rightarrow$ $\mathcal{C}(X)$ is a continuous surjection and $S \circ \bar{T}=\widetilde{T} \circ S$.

Proof. We first show the continuity of $S: \mathcal{K}(X) \rightarrow \mathcal{C}(X)$ in the case that $X$ is a complete locally convex space. The completeness is only needed in order to ensure that $S(\mathcal{K}(X)) \subset \mathcal{C}(X)$. Let $K \subset X$ be a compact subset and let $\mathcal{V}\left(U_{1}, \ldots, U_{r}\right)$ be an open neighbourhood of $S(K)$. We select a sufficiently small absolutely convex 0-neighbourhood $W$ such that

$$
S(K)+W \subset \bigcup_{i=1}^{r} U_{i} \text { and } \exists x_{i} \in \operatorname{co}(K) \text { with } x_{i}+W \subset U_{i}, \quad i=1, \ldots, r .
$$

By compactness, we find a finite collection $\left\{y_{1}, \ldots, y_{m}\right\} \subset K$ such that

$$
K \subset \bigcup_{j=1}^{m} V_{j}, \text { where } V_{j}:=y_{j}+\frac{1}{2} W, \quad j=1, \ldots, m \text {. }
$$

Certainly, $V:=\mathcal{V}\left(V_{1}, \ldots, V_{m}\right)$ is a neighbourhood of $K$. If $K^{\prime} \in V$ then we have that $S\left(K^{\prime}\right) \subset S(K)+W \subset \bigcup_{i=1}^{r} U_{i}$. Moreover, given $i \in\{1, \ldots, r\}$, we find a finite family $F=\left\{z_{1}, \ldots, z_{n}\right\} \subset K$ such that $x_{i}$ is a convex combination of elements in $F$. In particular, since for each $l \in\{1, \ldots, n\}$ there are $j_{l} \in$ $\{1, \ldots, m\}, y_{j_{l}}^{\prime} \in K^{\prime}$, and $w_{l}, w_{l}^{\prime} \in W$ such that $z_{l}=y_{j_{l}}+\frac{1}{2} w_{l}$ and $y_{j_{l}}^{\prime}=$ $y_{j_{l}}+\frac{1}{2} w_{l}^{\prime}$, we obtain that $z_{l} \in K^{\prime}+W, l=1, \ldots, n$. This means that $x_{i} \in$ $S\left(K^{\prime}\right)+W$, that is, $S\left(K^{\prime}\right) \cap U_{i} \supset S\left(K^{\prime}\right) \cap\left(x_{i}+W\right) \neq \emptyset$, which shows the continuity of $S . S: \mathcal{K}(X) \rightarrow \mathcal{C}(X)$ is trivially surjective, and the equality $S \circ \bar{T}=\widetilde{T} \circ S$ follows from the linearity and continuity of $T$. If $X$ is a locally convex space, not necessarily complete, then we easily have that $\left.S\right|_{\mathcal{F}(X)}$ : $\mathcal{F}(X) \rightarrow \mathcal{C}(X)$ has dense range.

Theorem 2.2. If $T$ is a continuous linear operator on a complete locally convex space $X$, then the following assertions are equivalent:

(i) $T$ is Devaney chaotic;

(ii) $\bar{T}$ is Devaney chaotic;

(iii) $\widetilde{T}$ is Devaney chaotic.

Proof. (i) $\Rightarrow$ (ii): Since $T$ is Devaney chaotic, $T$ is weakly mixing ([16], Corollary 3$)$. Hence, by $[1,27,30], \bar{T}$ is topologically transitive. Moreover, it is easy to check that the set

$$
\left\{\left\{x_{1}, \ldots, x_{k}\right\}: k \geq 1 \text { and } x_{1}, \ldots, x_{k} \in \operatorname{Per}(T)\right\}
$$

is dense in $\mathcal{K}(X)$ (because $\operatorname{Per}(T)$ is dense in $X)$ and it is contained in $\operatorname{Per}(\bar{T})$. (ii) $\Rightarrow$ (iii): It is a direct application of Lemma 2.1 . 
(iii) $\Rightarrow$ (i): We easily see that $T$ is topologically transitive. Indeed, let $U, V \subset$ $X$ be nonempty open sets and consider the basic (non-empty) open sets

$$
U^{\prime}:=\mathcal{V}(U) \cap \mathcal{C}(X) \quad \text { and } \quad V^{\prime}:=\mathcal{V}(V) \cap \mathcal{C}(X)
$$

in $\mathcal{C}(X)$. Since $\widetilde{T}$ is topologically transitive, there are $n \geq 0$ and $K^{\prime} \in U^{\prime}$ so that $\widetilde{T}^{n}\left(K^{\prime}\right) \in V^{\prime}$. Hence, there is $x \in K^{\prime} \subset U$ such that $T^{n} x \in V$. To show the density of the set of periodic points, by hypothesis there is $K \in U^{\prime}$ which is periodic for $\widetilde{T}$, i.e., $\widetilde{T}^{n}(K)=K$ for a certain $n \geq 1$. Since $K$ is nonempty, convex and compact, the Schauder-Tychonoff fixed point theorem ([33], Theorem 5.28) gives us a point $y \in U \cap \operatorname{Per}(T)$.

Remark 2.3. In the previous proof, the only implication where we used the fact that $X$ is complete was (ii) $\Rightarrow$ (iii). The implication (i) $\Rightarrow$ (ii) holds on an arbitrary topological vector space and the equivalence (i) $\Leftrightarrow$ (iii) holds on an arbitrary locally convex space.

Let us also mention that both implications

$$
\text { "T Devaney chaotic } \Rightarrow \bar{T} \text { Devaney chaotic" }
$$

and

$$
\text { "T Devaney chaotic } \Rightarrow T \text { Devaney chaotic" }
$$

are false (in general) in the context of nonlinear dynamics, that is, dynamics of continuous maps on compact metric spaces. See Remark 13 and Theorem 14 in $[18]$.

We recall that a family $\mathcal{A} \subset 2^{\mathbb{Z}_{+}}$is a collection of sets such that, if $A \in \mathcal{A}, B \subset \mathbb{Z}_{+}$, and $A \subset B$, then $B \in \mathcal{A}$. A family $\mathcal{A}$ is a filter if, in addition, given any $A, B \in \mathcal{A}$, we have that $A \cap B \in \mathcal{A}$. A family $\mathcal{A}$ is proper if $\emptyset \notin \mathcal{A}$ (equivalently, $\mathcal{A} \neq 2^{\mathbb{Z}_{+}}$). For a dynamical system $(X, T)$ a relevant family is

$$
\mathcal{N}_{T}:=\left\{A \subset \mathbb{Z}_{+}: \exists U, V \subset X \text { open and non-empty with } N_{T}(U, V) \subset A\right\} .
$$

With this terminology, $(X, T)$ is topologically transitive if and only if $\mathcal{N}_{T}$ is a proper family, and the weak mixing property is equivalent to the fact that $\mathcal{N}_{T}$ is a proper filter by a classical result of Furstenberg [14]. Given a family $\mathcal{A}$, we say that a dynamical system is $\mathcal{A}$-transitive if $\mathcal{N}_{T} \subset \mathcal{A}$ (that is, if $N_{T}(U, V) \in \mathcal{A}$ for each pair of nonempty open sets $\left.U, V \subset X\right)$. A trivial observation is that, given two families $\mathcal{A}$ and $\mathcal{B}$ such that $\mathcal{A} \subset \mathcal{B}$, if a dynamical system $(X, T)$ is $\mathcal{A}$-transitive, then it is $\mathcal{B}$-transitive. A thorough recent study of $\mathcal{A}$-transitivity in linear dynamics for several families $\mathcal{A}$ can be found in [8]. In this section we are interested in dynamical systems that are, at least, weakly mixing, in order to pass properties from the system to the corresponding hyperspace. Thus, by Furstenberg's result mentioned above, weakly mixing systems that are $\mathcal{A}$-transitive for a certain family $\mathcal{A}$ turn out to satisfy that $\mathcal{A}$ contains the proper filter $\mathcal{N}_{T}$. This is the reason why, for the rest of the section, we will concentrate on $\mathcal{A}$-transitivity for a proper filter $\mathcal{A}$. The equivalence of properties (i) and (ii) in the following theorem was given in [13] for general dynamical systems, using the arguments of $[1,27,30]$. 
Theorem 2.4. If $\mathcal{A}$ is a proper filter and $T$ is a continuous linear operator on a locally convex space $X$, then the following assertions are equivalent:

(i) $T$ is $\mathcal{A}$-transitive;

(ii) $\bar{T}$ is $\mathcal{A}$-transitive;

(iii) $\widetilde{T}$ is $\mathcal{A}$-transitive.

Proof. (i) $\Rightarrow$ (iii): We know that $\left.\bar{T}\right|_{\mathcal{F}(X)}$ is $\mathcal{A}$-transitive (see [13]) and, by Lemma 2.1 , we obtain that $\widetilde{T}$ is $\mathcal{A}$-transitive.

(iii) $\Rightarrow$ (i): Let $U, V \subset X$ be nonempty open sets. Then, $U^{\prime}:=\mathcal{V}(U) \cap \mathcal{C}(X)$ and $V^{\prime}:=\mathcal{V}(V) \cap \mathcal{C}(X)$ are nonempty open sets in $\mathcal{C}(X)$. By hypothesis, $N_{\widetilde{T}}\left(U^{\prime}, V^{\prime}\right) \in \mathcal{A}$, which implies that $N_{T}(U, V) \in \mathcal{A}$ since $N_{T}(U, V) \supset$ $N_{\widetilde{T}}\left(U^{\prime}, V^{\prime}\right)$.

We recall that a strictly increasing sequence $\left(n_{j}\right)_{j \in \mathbb{N}}$ of positive integers is syndetic if it has bounded gaps, that is,

$$
\sup _{j \in \mathbb{N}}\left(n_{j+1}-n_{j}\right)<\infty .
$$

A dynamical system $(X, T)$ is said to be topologically ergodic if for any pair $U, V \subset X$ of nonempty open sets, there is a syndetic sequence $\left(n_{j}\right)$ in $\mathbb{N}$ such that $T^{n_{j}}(U) \cap V \neq \emptyset$ for all $j \in \mathbb{N}$. A subset $A \subset \mathbb{Z}_{+}$is thickly syndetic if, for each $N \in \mathbb{N}$, the set $\left\{j \in \mathbb{Z}_{+}:\{j, j+1, \ldots, j+N\} \subset A\right\}$ is syndetic. Let us denote by $\mathcal{A}_{t s}$ the family of thickly syndetic sets, which is clearly a proper filter. Also, $\mathcal{A}_{c f}$ is the (proper) filter of co-finite subsets of $\mathbb{Z}_{+}$. For a general dynamical system $(X, T), \mathcal{A}_{c f}$-transitivity is equivalent to the mixing property. We also know (see the exercises in [17, Chapter 2]) that topologically ergodic operators are $\mathcal{A}_{t s}$-transitive. In particular, if $T_{1}$ is a topologically ergodic operator and $T_{2}$ is a weakly mixing operator, then $T_{1} \times T_{2}$ is weakly mixing (see $[15,31]$ ). Thus we immediately deduce the following result from Theorem 2.4.

Corollary 2.5. If $T$ is a continuous linear operator on a locally convex space $X$, then the following assertions are equivalent:

(i) $T$ is topologically ergodic (respectively, mixing);

(ii) $\bar{T}$ is topologically ergodic (respectively, mixing);

(iii) $\widetilde{T}$ is topologically ergodic (respectively, mixing).

Remark 2.6. The implication (i) $\Rightarrow$ (ii) in the above result for topological ergodicity is not always true in the case $T$ is a continuous map on a compact metric space $X$. Indeed, if (ii) holds, then $\bar{T}$ is topologically transitive, and so $T$ is necessarily weakly mixing. Thus, if $T$ is topologically ergodic but is not weakly mixing, then (i) holds but (ii) does not. Examples of such maps are irrational rotations on the unit circle. 


\section{Li-Yorke chaos}

Given a metric space $X$ and a continuous map $T: X \rightarrow X$, a pair $(x, y) \in X \times X$ is called a Li-Yorke pair for $T$ if

$$
\liminf _{n \rightarrow \infty} d\left(T^{n} x, T^{n} y\right)=0 \text { and } \quad \limsup _{n \rightarrow \infty} d\left(T^{n} x, T^{n} y\right)>0 .
$$

The map $T$ is said to be Li-Yorke chaotic if there exists an uncountable set $S$ (a scrambled set for $T$ ) such that $(x, y)$ is a Li-Yorke pair for $T$ whenever $x$ and $y$ are distinct points in $S$.

For studies of this notion of chaos in the context of continuous linear operators on Fréchet spaces, we refer the reader to the recent works $[5,6,21$, $22]$.

If a continuous linear operator $T$ on a Fréchet space $X$ is Li-Yorke chaotic, then so are $\bar{T}$ and $\widetilde{T}$, simply because the dynamical system $(X, T)$ can be regarded as a subsystem of the dynamical systems $(\mathcal{K}(X), \bar{T})$ and $(\mathcal{C}(X), \widetilde{T})$ by means of the isometric embedding

$$
x \in X \mapsto\{x\} \in \mathcal{C}(X) \subset \mathcal{K}(X) .
$$

A partial converse will be obtained below.

Lemma 3.1. Let $T$ be a continuous linear operator on a Fréchet space $X$. If $\bar{T}$ has a Li-Yorke pair, then there is a residual subset $Z$ of $X$ such that $\operatorname{Orb}(x, T)$ is unbounded for every $x \in Z$.

Proof. In view of the Banach-Steinhaus theorem, it is enough to prove that the sequence $\left(T^{n}\right)$ is not equicontinuous. By hypothesis, there is a Li-Yorke pair $(A, B)$ for $\bar{T}$. By definition, there is an increasing sequence $\left(p_{k}\right)$ of positive integers such that

$$
d_{H}\left(T^{p_{k}}(A), T^{p_{k}}(B)\right) \rightarrow 0 \quad \text { as } k \rightarrow \infty,
$$

and there are an $\varepsilon>0$ and an increasing sequence $\left(q_{k}\right)$ of positive integers such that

$$
d_{H}\left(T^{q_{k}}(A), T^{q_{k}}(B)\right) \geq \varepsilon \quad \text { for all } k \in \mathbb{N} .
$$

Fix $\delta>0$. It is enough to prove that there are $x \in X$ and $m \in \mathbb{N}$ such that

$$
d(x, 0)<\delta \quad \text { and } \quad d\left(T^{m} x, 0\right) \geq \varepsilon .
$$

By (3.1), we may find $j \in \mathbb{N}$ such that

$$
d_{H}\left(T^{p_{j}}(A), T^{p_{j}}(B)\right)<\delta .
$$

We select $k \in \mathbb{N}$ such that $q_{k}>p_{j}$. By (3.2), we may assume without loss of generality that there exists $a \in A$ such that

$$
d\left(T^{q_{k}} a, T^{q_{k}}(B)\right) \geq \varepsilon .
$$

By (3.3), $d\left(T^{p_{j}} a, T^{p_{j}}(B)\right)<\delta$, and so there exists $b \in B$ with $d\left(T^{p_{j}} a, T^{p_{j}} b\right)<$ $\delta$. Put $x:=T^{p_{j}}(a-b)$ and $m:=q_{k}-p_{j}$. Then

$$
d(x, 0)=d\left(T^{p_{j}} a, T^{p_{j}} b\right)<\delta .
$$


Moreover, by (3.4), $d\left(T^{q_{k}} a, T^{q_{k}} b\right) \geq \varepsilon$, and therefore

$$
d\left(T^{m} x, 0\right)=d\left(T^{q_{k}-p_{j}} T^{p_{j}}(a-b), 0\right) \geq \varepsilon .
$$

Theorem 3.2. Let $T$ be a continuous linear operator on a Fréchet space $X$ and define

$$
N S(T):=\left\{x \in X:\left(T^{n} x\right)_{n \in \mathbb{Z}_{+}} \text {has a subsequence converging to } 0\right\} .
$$

If $\operatorname{span}(N S(T))$ is dense in $X$, then the following assertions are equivalent:

(i) $T$ is Li-Yorke chaotic;

(ii) $\bar{T}$ is Li-Yorke chaotic;

(iii) $\bar{T}$ has a Li-Yorke pair;

(iv) $\widetilde{T}$ is Li-Yorke chaotic;

(v) $\widetilde{T}$ has a Li-Yorke pair.

Proof. The implications (i) $\Rightarrow$ (ii) $\Rightarrow$ (iii) and (i) $\Rightarrow$ (iv) $\Rightarrow$ (v) $\Rightarrow$ (iii) are trivial. Let us prove that (iii) $\Rightarrow$ (i). By the Li-Yorke Chaos Criterion [6, Theorem 15], we need to show the existence of a bounded sequence $\left(a_{n}\right)$ in $\overline{\operatorname{span}(N S(T))}$ such that the sequence $\left(T^{n} a_{n}\right)$ is unbounded. By (iii) and Lemma 3.1, we may take a vector $a \in X$ such that $\operatorname{Orb}(a, T)$ is unbounded. Since $\operatorname{span}(N S(T))$ is dense in $X, a \in \overline{\operatorname{span}(N S(T))}$. Hence, it is enough to put $a_{n}:=a$ for all $n \in \mathbb{Z}_{+}$.

Corollary 3.3. Let $X$ be a Fréchet sequence space in which $\left(e_{n}\right)_{n \in \mathbb{Z}_{+}}$is a basis ([17], Section 4.1). Suppose that the unilateral weighted backward shift

$$
B_{w}\left(x_{1}, x_{2}, x_{3}, \ldots\right):=\left(w_{2} x_{2}, w_{3} x_{3}, w_{4} x_{4}, \ldots\right)
$$

is an operator on $X$. Then the following assertions are equivalent:

(i) $B_{w}$ is Li-Yorke chaotic;

(ii) $\overline{B_{w}}$ is Li-Yorke chaotic;

(iii) $\overline{B_{w}}$ has a Li-Yorke pair;

(iv) $\widetilde{B_{w}}$ is Li-Yorke chaotic;

(v) $\widetilde{B_{w}}$ has a Li-Yorke pair.

Proof. In this case, the set $N S\left(B_{w}\right)$ contains a dense subspace of $X$, namely the set of all sequences of finite support. Hence, the corollary follows from the theorem.

Classical Banach sequence spaces are $\ell_{p}, 1 \leq p<\infty$, and $c_{0}$. In these particular cases, it was proved in [5] that $B_{w}$ is Li-Yorke chaotic if and only if

$$
\sup \left\{\left|w_{n} \cdot \ldots \cdot w_{m}\right|: n \in \mathbb{Z}_{+}, m>n\right\}=\infty .
$$

Therefore, we can offer a full characterization in terms of the weight sequence in this context. 
Corollary 3.4. Let $X:=\ell_{p}(1 \leq p<\infty)$ or $X:=c_{0}$, and let $w=\left(w_{n}\right)_{n \in \mathbb{Z}_{+}}$ be a bounded sequence of weights. Then properties (i)-(v) in Corollary 3.3 are also equivalent to:

(vi) $\sup \left\{\left|w_{n} \cdot \ldots \cdot w_{m}\right|: n \in \mathbb{Z}_{+}, m>n\right\}=\infty$.

We now turn our attention to bilateral weighted forward shifts.

Corollary 3.5. Let $X$ be a Fréchet sequence space over $\mathbb{Z}$ in which $\left(e_{n}\right)_{n \in \mathbb{Z}}$ is a basis ([17], Section 4.1). Suppose that the bilateral weighted forward shift

$$
F_{w}\left(\left(x_{n}\right)_{n \in \mathbb{Z}}\right):=\left(w_{n} x_{n-1}\right)_{n \in \mathbb{Z}}
$$

is an operator on $X$. Then the following assertions are equivalent:

(i) $F_{w}$ is Li-Yorke chaotic;

(ii) $\overline{F_{w}}$ is Li-Yorke chaotic;

(iii) $\overline{F_{w}}$ has a Li-Yorke pair;

(iv) $\widetilde{F_{w}}$ is Li-Yorke chaotic;

(v) $\widetilde{F_{w}}$ has a Li-Yorke pair.

Proof. It is enough to prove that (iii) $\Rightarrow$ (i). So, assume that there is a LiYorke pair $(A, B)$ for $\overline{F_{w}}$. We claim that

$$
\left(w_{1} \cdot \ldots \cdot w_{n} e_{n}\right)_{n \in \mathbb{Z}_{+}} \text {has a subsequence converging to zero. }
$$

Indeed, suppose that this is false. Without loss of generality, we may assume that there exists $x \in B$ such that $x \notin A$. Since $(A, B)$ is a Li-Yorke pair for $\overline{F_{w}}$, there are an increasing sequence $\left(n_{k}\right)$ in $\mathbb{Z}_{+}$and a sequence $\left(x^{(k)}\right)$ in $A$ such that

$$
\left(F_{w}\right)^{n_{k}}\left(x^{(k)}-x\right) \rightarrow 0 \text { as } k \rightarrow \infty .
$$

By passing to a subsequence, if necessary, we may assume that $\left(x^{(k)}\right)$ converges to a certain $y \in A$. Since the coordinate functionals are continuous, (3.6) implies that

$$
\left(x_{m}^{(k)}-x_{m}\right)\left(w_{m+1} \cdot \ldots \cdot w_{m+n_{k}}\right) e_{m+n_{k}} \rightarrow 0 \text { as } k \rightarrow \infty,
$$

for every $m \in \mathbb{Z}$. Since we are assuming that (3.5) is false, this implies that

$$
x_{m}^{(k)} \rightarrow x_{m} \text { as } k \rightarrow \infty,
$$

for every $m \in \mathbb{Z}$. Thus, $x=y \in A$, a contradiction. This proves that $(3.5)$ holds. Since (3.5) implies that $e_{n} \in N S\left(F_{w}\right)$ for all $n \in \mathbb{Z}$, Theorem 3.2 guarantees that $F_{w}$ is Li-Yorke chaotic.

\section{Remarks on topological transitivity and mixing properties}

We conclude the paper by generalizing some results on transitivity and weak mixing properties for hyperspace linear dynamics.

Banks [1], Liao, Wang and Zhang [27], and Peris [30] independently proved that the equivalences of the first three properties in the following theorem are true in the case $T$ is any continuous map and $X$ is any topological space (the equivalence (i) $\Leftrightarrow$ (ii) was first proved by Bauer and Sigmund [2] 
in the case $X$ is a compact metric space). The fact that (ii) $\Rightarrow$ (iv) was shown by Herzog and Lemmert for Banach spaces in [20]. The implication (v) $\Rightarrow(\mathrm{i})$ was provided in [30]. For the sake of completeness we will include a proof of the equivalence of property (i) with properties (iv) and (v) for locally convex spaces.

Theorem 4.1. If $T$ is a continuous linear operator on a topological vector space $X$, then the following assertions are equivalent:

(i) $T$ is weakly mixing;

(ii) $\bar{T}$ is weakly mixing;

(iii) $\bar{T}$ is topologically transitive.

If, in addition, $X$ is locally convex, then these assertions are also equivalent to the following:

(iv) $\widetilde{T}$ is weakly mixing;

(v) $\widetilde{T}$ is topologically transitive.

Proof. Let us assume that $X$ is a locally convex space.

(i) $\Rightarrow$ (iv): We know that $\left.\bar{T}\right|_{\mathcal{F}(X)}$ is weakly mixing and, by Lemma 2.1, we obtain that $\widetilde{T}$ is weakly mixing too, since $\left.S\right|_{\mathcal{F}(X)}: \mathcal{F}(X) \rightarrow \mathcal{C}(X)$ is a continuous map with dense range and $\left.\left.S\right|_{\mathcal{F}(X)} \circ \bar{T}\right|_{\mathcal{F}(X)}=\left.\widetilde{T} \circ S\right|_{\mathcal{F}(X)}$.

(iv) $\Rightarrow(\mathrm{v})$ : Obvious.

(v) $\Rightarrow$ (i): Let $U, V_{1}, V_{2} \subset X$ be nonempty open sets. It is enough to prove that there exists $n \geq 0$ such that

$$
T^{n}(U) \cap V_{1} \neq \emptyset \quad \text { and } \quad T^{n}(U) \cap V_{2} \neq \emptyset
$$

([17], Proposition 1.52). For this purpose, consider the basic open sets

$$
U^{\prime}:=\mathcal{V}(U) \cap \mathcal{C}(X) \quad \text { and } \quad V^{\prime}:=\mathcal{V}\left(V_{1}, V_{2}, X\right) \cap \mathcal{C}(X)
$$

in $\mathcal{C}(X) . U^{\prime}$ is clearly nonempty, and so is $V^{\prime}$ since any segment joining a point in $V_{1}$ with a point in $V_{2}$ belongs to $V^{\prime}$. Since $\widetilde{T}$ is topologically transitive, there are $n \geq 0$ and $K \in U^{\prime}$ so that $\widetilde{T}^{n}(K) \in V^{\prime}$. Hence, there are $x_{1}, x_{2} \in K \subset U$ such that $T^{n} x_{1} \in V_{1}$ and $T^{n} x_{2} \in V_{2}$.

De la Rosa and Read [11] solved a fundamental problem in linear dynamics by constructing hypercyclic operators on certain Banach spaces which are not weakly mixing. By modifying their construction, Bayart and Matheron [3] were able to construct such operators on classical Banach spaces, like $\ell^{p}(1 \leq p<\infty)$ and $c_{0}$. In view of Theorem 4.1 , such an operator $T$ is topologically transitive but $\bar{T}$ and $\widetilde{T}$ are not.

If $X$ is a separable $F$-space, it follows from well-known properties of hyperspaces that $\mathcal{K}(X)$ is a separable completely metrizable space without isolated points. Therefore, by Birkhoff's transitivity theorem, the topological transitivity of a continuous map $S: \mathcal{K}(X) \rightarrow \mathcal{K}(X)$ is equivalent to the existence of a point (or a residual set of points) with dense orbit under $S$. Moreover, in the case $X$ is a separable Fréchet space, the same is true for the space $\mathcal{C}(X)$. Indeed, the separability of $\mathcal{C}(X)$ follows immediately from the 
separability of $\mathcal{K}(X)$. Since $\mathcal{C}(X)$ is readily seen to be closed in $\mathcal{K}(X), \mathcal{C}(X)$ is also completely metrizable. In order to show that $\mathcal{C}(X)$ has no isolated point, fix $K \in \mathcal{C}(X)$ and let $U^{\prime}:=\mathcal{V}\left(U_{1}, \ldots, U_{r}\right) \cap \mathcal{C}(X)$ be a basic neighborhood of $K$ in $\mathcal{C}(X)$. Let $W$ be an open convex neighborhood of 0 in $X$ such that

$$
K+W \subset U_{1} \cup \ldots \cup U_{r} .
$$

Since $K \neq K+W$, we may choose vectors $x_{i} \in(K+W) \cap U_{i}$, for $i \in$ $\{1, \ldots, r\}$, so that at least one of the $x_{i}$ 's does not belong to $K$. Hence, the set $\operatorname{co}\left(\left\{x_{1}, \ldots, x_{r}\right\}\right)$ belongs to $U^{\prime}$ and is different from $K$. By combining these remarks with Theorem 4.1, we obtain:

Corollary 4.2. If $T$ is a continuous linear operator on a separable $F$-space $X$, then the following assertions are equivalent:

(i) $T$ is weakly mixing;

(ii) $\bar{T}$ has a point (a residual set of points) with dense orbit.

Moreover, if $X$ is a separable Fréchet space, then these assertions are also equivalent to

(iii) $\widetilde{T}$ has a point (a residual set of points) with dense orbit.

Herzog and Lemmert [20] considered continuous linear operators $T$ : $X \rightarrow X$ with the following property:

- (P) For any increasing sequence $\left(n_{k}\right)$ of natural numbers, there exists $x \in X$ such that $\overline{\left\{x, T^{n_{1}} x, T^{n_{2}} x \ldots\right\}}=X$.

They proved that property $(\mathrm{P})$ implies properties (ii) and (iii) in Corollary 4.2 in the case $X$ is a separable Banach space. On the other hand, a result due to Bès and Peris [9] tells us that the following properties are equivalent in the case $X$ is a separable $F$-space:

- (a) $T$ is weakly mixing;

- (b) There is an increasing sequence $\left(m_{k}\right)$ of natural numbers such that for any subsequence $\left(n_{k}\right)$ of $\left(m_{k}\right)$, there exists $x \in X$ with

$$
\overline{\left\{x, T^{n_{1}} x, T^{n_{2}} x \ldots\right\}}=X .
$$

- (c) $T$ satisfies the Hypercyclicity Criterion.

The Hypercyclicity Criterion is a very useful sufficient condition for hypercyclicity (see [17], Chapter 3). Obviously, property (P) implies property (b), so that it implies property (i) in Corollary 4.2. However, (P) is strictly stronger than (i) even in Hilbert spaces (see Salas [34]). Later, Peris [30] improved the result of Herzog and Lemmert by establishing the equivalence of conditions (i), (iii) and (v) in Theorem 4.1, and the Hypercyclicity Criterion, in the case $X$ is a separable Fréchet space.

\section{Acknowledgement}

This work was partially done on a visit of the first author to the Departament de Matemàtica Aplicada at Universitat Politècnica de València (Spain). The first author is very grateful for the hospitality and support. We thank the 
referee for valuable suggestions that produced a better presentation of the paper.

\section{References}

[1] J. Banks, Chaos for induced hyperspace maps, Chaos Solitons Fractals 25 (2005), no. 3, 681-685.

[2] W. Bauer and K. Sigmund, Topological dynamics of transformations induced on the space of probability measures, Monatsh. Math. 79 (1975), 81-92.

[3] F. Bayart and É. Matheron, Hypercyclic operators failing the hypercyclicity criterion on classical Banach spaces, J. Funct. Anal. 250 (2007), no. 2, 426441.

[4] F. Bayart and É. Matheron, Dynamics of Linear Operators, Cambridge University Press, Cambridge, 2009.

[5] T. Bermúdez, A. Bonilla, F. Martínez-Giménez and A. Peris, Li-Yorke and distributionally chaotic operators, J. Math. Anal. Appl. 373 (2011), no. 1, 8393.

[6] N. C. Bernardes Jr., A. Bonilla, V. Müller and A. Peris, Li-Yorke chaos in linear dynamics, Ergodic Theory Dynam. Systems 35 (2015), no. 6, 1723-1745.

[7] N. C. Bernardes Jr. and R. M. Vermersch, Hyperspace dynamics of generic maps of the Cantor space, Canad. J. Math. 67 (2015), no. 2, 330-349.

[8] J. Bès, Q. Menet, A. Peris and Y. Puig, Strong transitivity properties for operators. Preprint (arXiv:1703.03724).

[9] J. Bès and A. Peris, Hereditarily hypercyclic operators, J. Funct. Anal. 167 (1999), no. 1, 94-112.

[10] J. Bonet, L. Frerick, A. Peris, and J. Wengenroth, Transitive and hypercyclic operators on locally convex spaces, Bull. London Math. Soc. 37 (2005), 254264.

[11] M. de la Rosa and C. Read, A hypercyclic operator whose direct sum $T \oplus T$ is not hypercyclic, J. Operator Theory 61 (2009), no. 2, 369-380.

[12] A. Fedeli, On chaotic set-valued discrete dynamical systems, Chaos Solitons Fractals 23 (2005), no. 4, 1381-1384.

[13] H. Fu and Z. Xing, Mixing properties of set-valued maps on hyperspaces via Furstenberg families, Chaos Solitons Fractals 45 (2012), no. 4, 439-443.

[14] H. Furstenberg, Disjointness in ergodic theory, minimal sets, and a problem in Diophantine approximation, Math. Systems Theory 1 (1967), 1-49.

[15] S. Grivaux, Hypercyclic operators, mixing operators, and the bounded steps problem, J. Operator Theory 54 (2005), 147--168.

[16] K.-G. Grosse-Erdmann and A. Peris, Weakly mixing operators on topological vector spaces, Rev. R. Acad. Cienc. Exactas Fís. Nat. Ser. A Math. RACSAM 104 (2010), no. 2, 413-426.

[17] K.-G. Grosse-Erdmann and A. Peris, Linear Chaos, Universitext, SpringerVerlag, 2011.

[18] J. L. G. Guirao, D. Kwietniak, M. Lampart, P. Oprocha and A. Peris, Chaos on hyperspaces, Nonlinear Anal. 71 (2009), no. 1-2, 1-8. 
[19] P. Hernández, J. King and H. Méndez, Compact sets with dense orbit in $2^{X}$, Topology Proc. 40 (2012), 319-330.

[20] G. Herzog and R. Lemmert, On universal subsets of Banach spaces, Math. Z. 229 (1998), no. 4, 615-619.

[21] B. Hou, G. Tian and L. Shi, Some dynamical properties for linear operators, Illinois J. Math. 53 (2009), no. 3, 857-864.

[22] B. Hou, G. Tian and S. Zhu, Approximation of chaotic operators, J. Operator Theory 67 (2012), no. 2, 469-493.

[23] A. Illanes and S. B. Nadler Jr., Hyperspaces: Fundamentals and Recent Advances, Marcel Dekker, Inc., New York, 1999.

[24] J. Kupka, On Devaney chaotic induced fuzzy and set-valued dynamical systems, Fuzzy Sets and Systems 177 (2011), no. 1, 34-44.

[25] D. Kwietniak and P. Oprocha, Topological entropy and chaos for maps induced on hyperspaces, Chaos Solitons Fractals 33 (2007), no. 1, 76-86.

[26] J. Li, K. Yan and X. Ye, Recurrence properties and disjointness on the induced spaces, Discrete Cont. Dynam. Syst. 35 (2015), no. 3, 1059-1073.

[27] G. Liao, L. Wang and Y. Zhang, Transitivity, mixing and chaos for a class of set-valued mappings, Science in China: Ser. A Math. 49 (2006), no. 1, 1-8.

[28] H. Liu, E. Shi and G. Liao, Sensitivity of set-valued discrete systems, Nonlinear Anal. 71 (2009), no. 12, 6122-6125.

[29] H. Liu, F. Lei and L. Wang, Li-Yorke sensitivity of set-valued discrete systems, J. Appl. Math. (2013). Article number: 260856.

[30] A. Peris, Set-valued discrete chaos, Chaos Solitons Fractals 26 (2005), no. 1, 19-23.

[31] A. Peris and L. Saldivia, Syndetically hypercyclic operators, Integral Equations Operator Theory 51 (2005), 275-281.

[32] H. Román-Flores, A note on transitivity in set-valued discrete systems, Chaos Solitons Fractals 17 (2003), no. 1, 99-104.

[33] W. Rudin, Functional Analysis, Second Edition, International Series in Pure and Applied Mathematics, McGraw-Hill, Inc., New York, 1991.

[34] H. Salas, A hypercyclic operator whose adjoint is also hypercyclic, Proc. Amer. Math. Soc. 112 (1991), no. 3, 765-770.

[35] S. Shkarin, Hypercyclic operators on topological vector spaces, J. Lond. Math. Soc. 86 (2012), 195-213.

[36] Y. Wang and G. Wei, Characterizing mixing, weak mixing and transitivity of induced hyperspace dynamical systems, Topology Appl. 155 (2007), no. 1, 5668.

[37] Y. Wang, G. Wei and W. H. Campbell, Sensitive dependence on initial conditions between dynamical systems and their induced hyperspace dynamical systems, Topology Appl. 156 (2009), no. 4, 803-811.

[38] Y. Wu, X. Xue and D. Ji, Linear transitivity on compact connected hyperspace dynamics, Dynam. Systems Appl. 21 (2012), no. 4, 523-534.

[39] X. Wu, J. Wang and G. Chen, F-sensitivity and multi-sensitivity of hyperspatial dynamical systems, J. Math. Anal. Appl. 429 (2015), no. 1, 16-26. 
N. C. Bernardes Jr.

Departamento de Matemática Aplicada, Instituto de Matemática, Universidade Federal do Rio de Janeiro,

Caixa Postal 68530, Rio de Janeiro,

RJ, 21945-970, Brasil

e-mail: bernardes@im.ufrj.br
A. Peris
Institut Universitari de Matemàtica Pura i Aplicada, Universitat Politècnica de València, Edifici 8E, Cub F, 4a Planta, 46022 València, Spain
e-mail: aperis@mat.upv.es
F. Rodenas
Institut Universitari de Matemàtica Pura i Aplicada, Universitat Politècnica de València, Edifici 8E, Cub F, 4a Planta, 46022 València, Spain
e-mail: frodenas@mat.upv.es 